\title{
Backscatter Coefficient as an Attribute for the Classification of Full-waveform Airborne Laser Scanning Data in Urban Areas
}

\author{
Cici Alexander ${ }^{1}$, Kevin Tansey ${ }^{1}$, Jörg Kaduk ${ }^{1}$, David Holland ${ }^{2}$, Nicholas J. Tate ${ }^{1}$ \\ ${ }^{1}$ Department of Geography, University of Leicester \\ University Road, Leicester LE1 7RH, UK \\ ${ }^{2}$ Ordnance Survey, \\ Romsey Road, Southampton SO16 4GU, UK
}

\begin{abstract}
Airborne laser scanning (ALS) data are increasingly being used for land cover classification. The amplitudes of echoes from targets, available from full-waveform ALS data, have been found to be useful in the classification of land cover. However, the amplitude of an echo is dependent on various factors such as the range and incidence angle, which makes it difficult to develop a classification method which can be applied to full-waveform ALS data from different sites, scanning geometries and sensors. Additional information available from full-waveform ALS data, such as range and echo width, can be used to calibrate intensity values, and derive backscatter cross section. The backscatter cross section of a target is the physical cross sectional area of an idealised isotropic target, which has the same intensity as the selected target. The backscatter coefficient is the backscatter cross section per unit area. In this study, the amplitude, backscatter cross section and backscatter coefficient of echoes from ALS point cloud data collected from two different sites are analysed based on urban land cover classes. The application of decision tree classifiers developed using data from one study area on another demonstrates the advantage of using backscatter coefficient in classification methods, along with spatial attributes. It is shown that the accuracy of classification using backscatter coefficient (kappa coefficient 0.89) is higher than those using amplitude (kappa coefficient 0.67) or backscatter cross section (kappa coefficient 0.68 ) in the classification of urban areas. This attribute is especially useful for separating road and grass.
\end{abstract}

KEYWORDS: Classification; Laser scanning; Point Cloud; Comparison; LIDAR; Backscatter Coefficient 


\section{Introduction}

Airborne laser scanning (ALS) data are increasingly being used for land cover mapping. There are two distinct techniques used in ALS systems based on how the return signal is recorded - discrete return and full-waveform. Full-waveform ALS data give additional information about the objects in the path of the laser pulse, when compared to discrete return ALS data. In a full-waveform scanner, the return signal is the product of the transmitted laser pulse and the scattering function of the target or targets. The temporal shape of the return signal is often referred to as waveform. In the case of a single flat target perpendicular to the incident laser beam, the waveform is a smaller version of the transmitted signal in terms of shape. The waveform recording ALS systems record the entire timevarying power of the return signal from all illuminated surfaces. The waveform from multiple targets is complex, and represents the sum of the signals from the individual targets. It is often decomposed into components representing the individual targets. Many approaches to the interpretation of ALS data are based on Gaussian decomposition. Its underlying assumption is that the scattering properties of a cluster of targets can be described by a Gaussian function. An extended target could be described by a series of Gaussian functions, where each pulse represents a cluster of targets too close to be differentiated. This method gives estimates of the location and scattering properties of the targets (Hofton et al., 2000; Mallet and Bretar, 2009; Wagner et al., 2006).

Amplitude, echo width and the number of returns are the additional information obtained from fullwaveform data, which have been successfully used for classification. The maximum amplitude is a measure of the strength of the echo. The echo width could refer either to the full width at half the maximum (FWHM) amplitude, or the standard deviation, of the pulse in the Gaussian decomposition. The main methods used by the classification studies using full-waveform ALS data are decision trees (Ducic et al., 2006; Rutzinger et al., 2008) and support vector machines (Bretar et al., 2009; Mallet et al., 2008). Ducic et al. (2006) used a decision tree to classify ALS points into vegetation (trees and shrubs) and non-vegetation (grass, roof and road) using attributes from fullwaveform ALS data. In this study, although the points from grass and road could be separated based on amplitude, grass had similar range of amplitudes to building roofs. Since their aim was to classify points without using elevation or relationship to adjacent points, grass was grouped with nonvegetation for generating the decision tree. Rutzinger et al. (2008) used a decision tree to classify points from full-waveform ALS data to detect tall vegetation - trees and shrubs, based on the homogeneity of echo width. Segment statistics were calculated by aggregating attributes such as amplitude and surface roughness for a training area. This was used to construct a decision tree to classify the ALS data. They achieved accuracies above $90 \%$ for the validation sites.

The amplitude values would vary for a given target depending on the flying height or elevation differences, even within a single dataset. Multi-temporal analysis using amplitude values, from different ALS systems, scan geometry and atmospheric conditions, would require the values to be converted to comparable measurements. The additional information provided by the decomposition of full-waveform ALS data is suitable for calibration, whereby amplitude and echo width are converted to values proportional to the surface reflectance of the target (Coren and Sterzai, 2006; Höfle and Pfeifer, 2007; Kaasalainen et al., 2007; Wagner et al., 2008b).

In radar remote sensing, the backscattering or backscatter cross section, also referred to as radar cross section is of the most interest. Since lidar (the technique used in laser scanning) is considered to be an extension of radar to shorter wavelengths, the radar cross section is often used to understand the characteristics of the return signal from laser scanning. The backscatter cross section of a target, usually represented by the Greek letter sigma $(\sigma)$, is equal to the physical cross sectional area of an idealised isotropic target, which has the same intensity as the selected target. It has dimensions of area, in $\mathrm{m}^{2}$ (Wagner et al., 2006; Woodhouse, 2006). 
The radar equation has been used to explain the strength of the return signal in ALS, and to derive the backscatter cross section. When measurements from different flight conditions and instruments are to be compared, the measured receiver power has to be converted into backscatter cross section. The backscatter cross section is derived from the range (distance from the sensor to the target), amplitude and width of an echo. The calibration constant is a single value that sums up the parameters that are constant for a particular flight taking into consideration the system and atmospheric parameters. The calibration constant can be estimated using reference targets with a known backscatter cross section, or a known surface reflectance at the wavelength of the sensor. Asphalt roads have been used for deriving the calibration constant based on an assumed or fieldmeasured reflectance value (Briese et al., 2008; Wagner et al., 2006). This is discussed further in section 3.3.

The backscatter cross section need not correspond to the actual cross sectional area of the target, and for a small discrete target, the value would remain the same with a decrease or increase in the illuminated area. However, for an extended target, like bare ground, the backscatter cross section would increase or decrease in proportion to the illuminated area. This makes it difficult to compare the cross sections of targets, with similar scattering properties, but different physical areas. The backscatter coefficient is a normalised measure of the backscatter cross section irrespective of the area of the footprint (Woodhouse, 2006).

Backscatter cross section and backscatter coefficient have been suggested as suitable values for describing the scattering properties of targets, and for calibration (Wagner et al., 2008b). However, they have not yet been analysed as potential attributes in the classification of urban areas. Even in the case of decision tree classifiers using amplitude and echo width, a method developed based on values from one study area has not been used to classify data with a different scanning geometry from another study area. In this study, amplitude, backscatter cross section and backscatter coefficient are compared for use as attributes in the classification of urban areas into vegetation, roads and building roofs. The amplitude values in one study area are calibrated to obtain the backscatter cross section and backscatter coefficient of each point in the training data. Amplitude, backscatter cross section and backscatter coefficient are used to construct decision trees for classifying the ALS points. The decision trees are then used to classify data points from the second study area to assess the influence of the flying height and scan angle on these attributes, and on the accuracy of classification.

Vegetation is subdivided into grass, shrubs and trees based on height $(<0.5 \mathrm{~m}, 0.5-2.5 \mathrm{~m}$ and $>2.5$ $\mathrm{m})$, and buildings are subdivided into those with flat and pitched roofs. The other attributes used for classification are echo width, normalised elevation, average and standard deviation of slopes, standard deviation of aspect, height variation and number of echoes. The classes and the derivation of spatial attributes are discussed in more detail in Alexander et al. (2008). The accuracies of the three classifications of the second study area are compared. Aerial photographs and a digital topographic map (OS MasterMap ${ }^{\circledR}$ ) are used only for analysis, and for creating training and reference data.

\section{Study Area and Datasets}

Full-waveform ALS datasets were collected from Bristol and Bournemouth using LiteMapper 5600 Airborne Lidar Terrain Mapping System, in August 2006 and June 2008, respectively. The LiteMapper system makes use of RIEGL LMS-Q560 laser scanner. The sensor used in this system emits laser pulses at a wavelength of $1550 \mathrm{~nm}$. The system has a laser beam divergence angle of $0.5 \mathrm{mrad}$, which produces a footprint diameter of approximately $0.5 \mathrm{~m}\left(1000 \times 0.5 \times 10^{-3}\right)$ on a target at nadir, 
from a flying height of $1 \mathrm{~km}$. The footprint diameter increases with the range or flying height, and the off-nadir footprints are elliptical. The two datasets differ in the heights from which data were collected, and the maximum scan angles.

In Bristol, data were captured at a height of approximately $950 \mathrm{~m}$ above ground level, at a speed of $65 \mathrm{~ms}^{-1}$ with a laser scanning pulse rate of $50 \mathrm{kHz}$. The maximum scan angle for the dataset was $22.5^{\circ}$ from nadir. The point density ranged from $0.5-0.8$ points $\mathrm{m}^{-2}$ per flight line. The average footprint diameter on the target at nadir with a laser beam divergence of $0.5 \mathrm{mrad}$ is approximately $0.475 \mathrm{~m}$. An area of $1 \mathrm{~km} \times 1 \mathrm{~km}$ was chosen from Easting 354000 to 355000 (longitude 2³9'50" W to $2^{\circ} 38^{\prime} 59^{\prime \prime} \mathrm{W}$ ) and Northing 178000 to 179000 (latitude $51^{\circ} 29^{\prime} 56^{\prime \prime} \mathrm{N}$ to $51^{\circ} 30^{\prime} 29^{\prime \prime} \mathrm{N}$ ), which includes a range of land use and land cover types. In addition to stands of trees, there are trees along the road, shrubs in gardens in the residential areas as well as grassland. The land use includes residential and institutional areas and agricultural land. Four flight strips, with overlaps, covered the whole study area.

The second study area is in Bournemouth (Figure 1), with an area of $0.5 \mathrm{~km} \times 0.5 \mathrm{~km}$ from Easting 408750 to 409250 (longitude $1^{\circ} 52^{\prime} 38^{\prime \prime}$ W to $1^{\circ} 52^{\prime} 13^{\prime \prime}$ W) and Northing 91500 to 92000 (latitude $50^{\circ} 43^{\prime} 23^{\prime \prime} \mathrm{N}$ to $\left.50^{\circ} 43^{\prime} 39^{\prime \prime} \mathrm{N}\right)$. It is a more complex site than the one in Bristol. Horseshoe common, in the middle of the study area, has a variety of trees on a sloping terrain. The road network is also complex with bridges and flyovers. The full-waveform data were collected from a height of approximately $300 \mathrm{~m}$ and a maximum scan angle of $30^{\circ}$. The study area was covered by the swath widths of seven flight lines. The footprint diameter on the target collected from a flying height of $300 \mathrm{~m}$ at nadir is $0.15 \mathrm{~m}$. The major axis of the elliptical footprint at the maximum scan angle of $30^{\circ}$ is about $15 \%$ longer, and the area is about $54 \%$ larger.

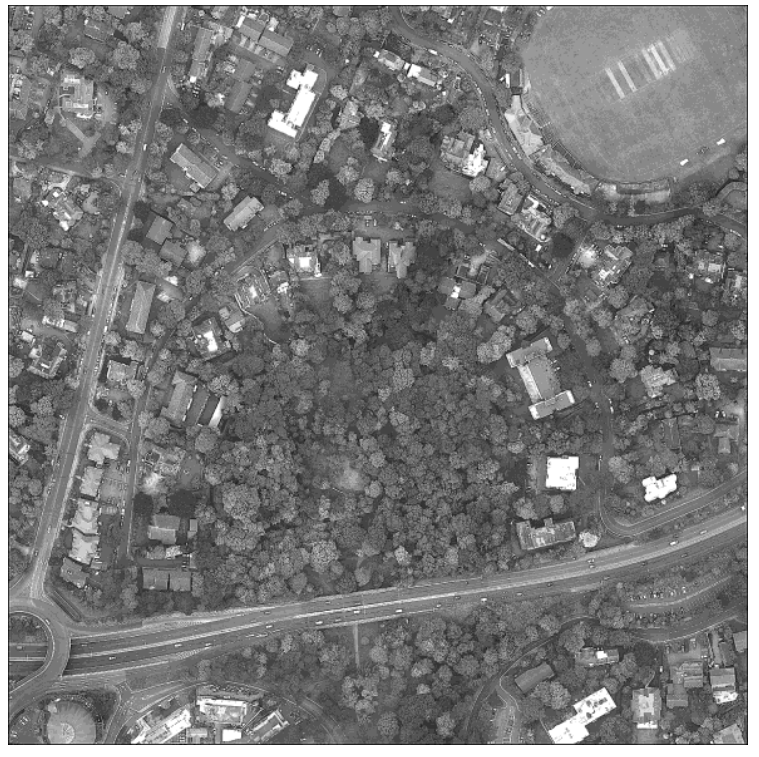

Figure 1. Aerial image of the study area in Bournemouth (Ordnance Survey @ Crown Copyright. All Rights Reserved)

\section{Methodology}

The calibration constants for the two study areas can be calculated based on assumptions about the reflectance of asphalt at the wavelength of the sensor. Wagner et al. (2006) derived the value of the calibration constant based on the assumption that an asphalt road has a reflectance of 0.2 at a wavelength of $1550 \mathrm{~nm}$. Briese et al. (2008) made use of a reflectometer developed by Riegl, to measure the reflectance of three surfaces of interest: two sections of asphalt road and one building 
roof. The Riegl reflectometer made use of a laser diode operating at $1550 \mathrm{~nm}$, producing a spot of approximately $0.15 \mathrm{~m}$ diameter at a distance of $1-1.5 \mathrm{~m}$. The reflectance of two Spectralon ${ }^{\circledR}$ reference targets, of reflectance $99 \%$ and $60 \%$, were measured using the reflectometer and used to calculate the reflectance of the areas of interest. In this case, the reflectance of asphalt at the said wavelength was found to be 0.25 . A reflectance of 0.25 is therefore used in this study.

Initially, the calibration constants for the two study areas are calculated. The backscatter cross sections are calculated based on this value. The backscatter cross sections for the two study areas would have been comparable if the flying height and footprint diameters were the same. Since this is not the case, the backscatter coefficients are calculated by normalising the backscatter cross sections with the area of the footprint. Decision tree classifiers are generated using amplitude, backscatter cross section and the backscatter coefficient derived from the training points in Bristol. These decision tree classifiers are then applied on the data from Bournemouth. The classifications are compared to determine whether the calibrated intensity values give advantages over amplitude, in terms of accuracy.

\subsection{Pre-processing}

The raw waveforms from Bristol and Bournemouth were decomposed using the commercial package RiAnalyze 560. RiWorld 560 was used to transform the data into WGS Cartesian coordinates. The dataset was transformed to British National Grid, using Grid InQuest (Quest, 2009), before further analysis. The point density ranged from $0.5-0.8$ points $\mathrm{m}^{-2}$ for each flight line in Bristol. All the echoes were used for the analysis, which along with the overlapping swath widths of the flight lines generated above 1000000 points giving an average point density of approximately 1 point $\mathrm{m}^{-2}$. The number of echoes denotes whether the point is one of a single, two, three or more hits of a single emitted pulse. $94.32 \%$ of the points were first echoes, $5.4 \%$ were second echoes, $0.28 \%$ third echoes and $0.06 \%$ fourth echoes. $88.97 \%$ of the points were single echoes, and only one point was a fifth echo.

The dataset from Bournemouth contained 12914815 points from seven flight lines, providing a point density of above 50 points $\mathrm{m}^{-2}$. The points had to be thinned to make a better comparison with the data from Bristol, which was approximately 1 point $\mathrm{m}^{-2}$. A point shapefile was created with points at $1 \mathrm{~m}$ spacing in a grid within the study area. Data points that were nearest to the grid points were selected. In the case of multiple echoes, all echoes with the same time stamp were also added to the selection. After thinning, there were 337198 points in an area of 250000 sq. $\mathrm{m}$ in the dataset in Bournemouth.

\subsection{Attributes for generating decision trees}

Amplitude, elevation, echo width, echo number and the number of echoes are the attributes of individual points extracted from the full-waveform data. The amplitude values of roads and trees are lower than those of grass, shrubs and buildings. Although there are overlaps in the values, amplitude is a useful attribute in separating road and grass. The normalised elevation is useful in separating buildings from the other classes, especially road and grass.

The spatial relationships between neighbouring points were derived based on a TIN, which was created from the elevation of all the points. The attributes used in the decision tree were average and standard deviation of slopes of TIN triangles attached to each point, standard deviation of aspect and height variation. 
The height of points from the ground can be useful in distinguishing different surfaces. With the use of additional attributes derived from the TIN, it is hoped that the classifier would work even with a rough digital terrain model (DTM). A rough DTM was therefore created from the elevation of ALS points, making use of the lowest point in a $10 \mathrm{~m}$ grid. A TIN was created from these points. The terrain elevation obtained from this TIN surface was subtracted from the elevation of each point to obtain an estimate of the normalised elevation.

\subsection{Calculation of the Calibration Constants}

The theoretical backscatter cross section of an echo at nadir is (Briese et al., 2008; Höfle and Pfeifer, 2007; Jelalian, 1991; Wagner et al., 2006):

$$
\sigma=\pi \rho R^{2} \beta^{2}
$$

where $\sigma$ is the backscatter cross section in $\mathrm{m}^{2}, \rho$ is the reflectance of the target surface, $R$ is the range (the distance from the sensor to the target) in $\mathrm{m}$ and $\beta$ is the laser beam divergence angle in radians.

From the radar equation, Wagner et al. (2006) derived the following equation for the 'apparent' cross section of each surface within the laser footprint:

$$
\sigma=C_{c a l} R^{4} P W
$$

where $C_{c a l}$ is the calibration constant, $P$ is the amplitude of the echo, $W$ is the echo width Assuming a reflectance of 0.25 for asphalt, the calibration constant can be calculated from the equations, [1] and [2] as:

$$
C_{c a l}=\frac{0.25 \pi \beta^{2}}{R^{2} P W}
$$

where $P$ is the amplitude and $W$ is the echo width of each point on the selected sections of asphalt road. The laser beam divergence angle $(\beta)$, is $0.5 \mathrm{mrad}$ for Riegl LMS-Q560. The width of the echo from the Riegl software corresponds to the FWHM of amplitude of the Gaussian pulse. This was converted to the standard deviation of the Gaussian using the formula (Weisstein, 2009):

$$
W=\frac{F W H M}{2 \sqrt{2 \ln 2}}
$$

Near-nadir points from asphalt road sections were selected from the study area in Bristol. The scan angle being almost $90^{\circ}$, almost horizontal sections were chosen to avoid the influence of incidence angle on the echo. Points with amplitude greater than 40 were removed so that points from white road markings were not included. There were 566 points from the nine road sections. The mean of the calculated calibration constants, $4.45 .10^{-6}$, was used for the calculation of backscatter cross section and backscatter coefficients. 


\subsection{Derivation of Backscatter Cross Sections and Backscatter Coefficients}

The backscatter cross sections $(\sigma$ ) for each echo were calculated using equation [2]. The values increase with the area of the laser beam footprint on the target. For a particular beam width, the area of the footprint changes with the flying height at nadir. The cross section per unit area, denoted as $\gamma$, is considered to be a better attribute for comparing the scattering characteristics of areaextensive targets which produce single echoes from different sensors and flight parameters (Ulaby et al., 1982; Wagner et al., 2008b). The cross section per unit illuminated area $\left(\sigma^{0}\right)$ is the most widely used parameter in radar remote sensing. However, this requires the incidence angle of each echo, and significant processing and modelling are necessary to estimate the local incidence angle on an inclined surface (Wagner et al., 2008b). Although the illuminated area changes with the incidence angle of the beam on a given surface, the scattering strength is often related to the cross section of the incoming beam for convenience (Schanda, 1986; Wagner et al., 2008b).

Cross sectional area of the incoming beam (Wagner et al., 2008b), A, is:

$$
A=\frac{\pi \cdot R^{2} \cdot \beta^{2}}{4}
$$

where $R$ is the distance from the sensor to the target in $\mathrm{m}$ and $\beta$ is the laser-beam divergence angle in radians.

The back scatter cross section per unit area of the incoming beam can be calculated as (Wagner et al., 2008b):

$$
\gamma=\frac{\sigma}{\mathrm{A}}
$$

Amplitude and the derived attributes, $\sigma$ and $\gamma$, were used to generate decision trees to classify the full-waveform dataset.

\subsection{Generation of Decision Trees for Classification}

Training polygons were manually delineated using the attributes of the ALS points, OS MasterMap and an aerial image into the six land cover classes. The attributes of points within the training polygons representing the different classes could now be analysed. The attributes - amplitude, echo width, number of returns, average and standard deviation of slopes, standard deviation of aspect, height variation and normalised elevation - of the dataset from Bristol were used to generate a decision tree for classification. Decision trees were generated, replacing the amplitude by the backscatter cross section as an attribute. This was repeated using the backscatter coefficient. These three decision trees, using amplitude, backscatter cross section and backscatter coefficient, were then used to classify the data from Bournemouth.

\subsection{Classification of ALS Points}

A TIN was created from the thinned data points in Bournemouth, using elevation as the attribute. This was converted to TIN triangles and intersected with the points, to derive additional spatial attributes. The thinned dataset from Bournemouth now had the following attributes for each received echo: unique identifier, time stamp, easting, northing, elevation, amplitude, echo number, number of echoes, range, scan angle and echo width. 
Ten sections of asphalt road were selected from the un-thinned Bournemouth dataset. The points selected were from road sections with scan angle from $89.5^{\circ}$ to $90.5^{\circ}$ and the range of heights less than $0.2 \mathrm{~m}$. There were 1898 points from the ten road sections. The mean of the calculated calibration constants, $17.4 \times 10^{-6}$, was used for the calculation of backscatter cross section and backscatter coefficients for each point in the dataset.

The three decision trees generated using the training data from Bristol were then used to classify the points. The attributes 'class' and 'node' were added to the existing attributes of each point. The points could now be displayed according to their class for visual analysis. The classification results using the three decision trees were compared, as discussed in the following section.

\subsection{Assessment of Accuracies}

Reference polygons belonging to the different classes were manually delineated using the attributes of the ALS points, and visual analysis of the aerial photograph and OS MasterMap. The points within these reference polygons were selected for assessing the accuracies of classification. There were 3 520 points in all, consisting of 1076 points from trees, 109 points from shrubs, 708 points from grass, 702 points from road, 399 points from flat roofs and 526 points from pitched roofs. The class of the manually referenced point was added as an attribute of a point before classification. The classification of points using the three decision trees were compared with the class of the reference data to get an estimate of the accuracies using the different attributes.

\section{Results}

The kappa coefficients, overall accuracies, average user's accuracies and average producer's accuracies of classification using the three decision trees are shown in Table 1. All the accuracies are the highest for the third classification using $\gamma$ as an attribute. The calibration constants in Bristol and Bournemouth were calculated as $4.45 \times 10^{-6}$ and $17.4 \times 10^{-6}$ respectively. These were used to calibrate the intensities of the datasets from the two study areas, and estimate the backscatter cross sections and backscatter coefficients.

Table 1: Kappa coefficients, overall accuracies, average user's accuracies and average producer's accuracies of the classifications for the three decision trees using amplitude, $\sigma$ and $\gamma$ as attributes

\begin{tabular}{|l|l|l|l|}
\hline & Amplitude & $\sigma$ & $\gamma$ \\
Kappa coefficient & 0.67 & 0.68 & 0.89 \\
Overall Accuracy & 73.44 & 74.52 & 91.53 \\
Average User's Accuracy & 82.23 & 68.24 & 90.36 \\
Average Producer's Accuracy & 78.93 & 76.70 & 91.11 \\
\hline
\end{tabular}

The classification using the decision tree with amplitude as an attribute is shown in Figure 2. A major misclassification is that of road at a higher elevation than the land below as a building (Figure 3). On visual analysis of the data, displayed by the assigned node number, the other major misclassifications are in the nodes using amplitude (Figure 4). The resulting leaf nodes are grass, road, trees and pitched roofs. 

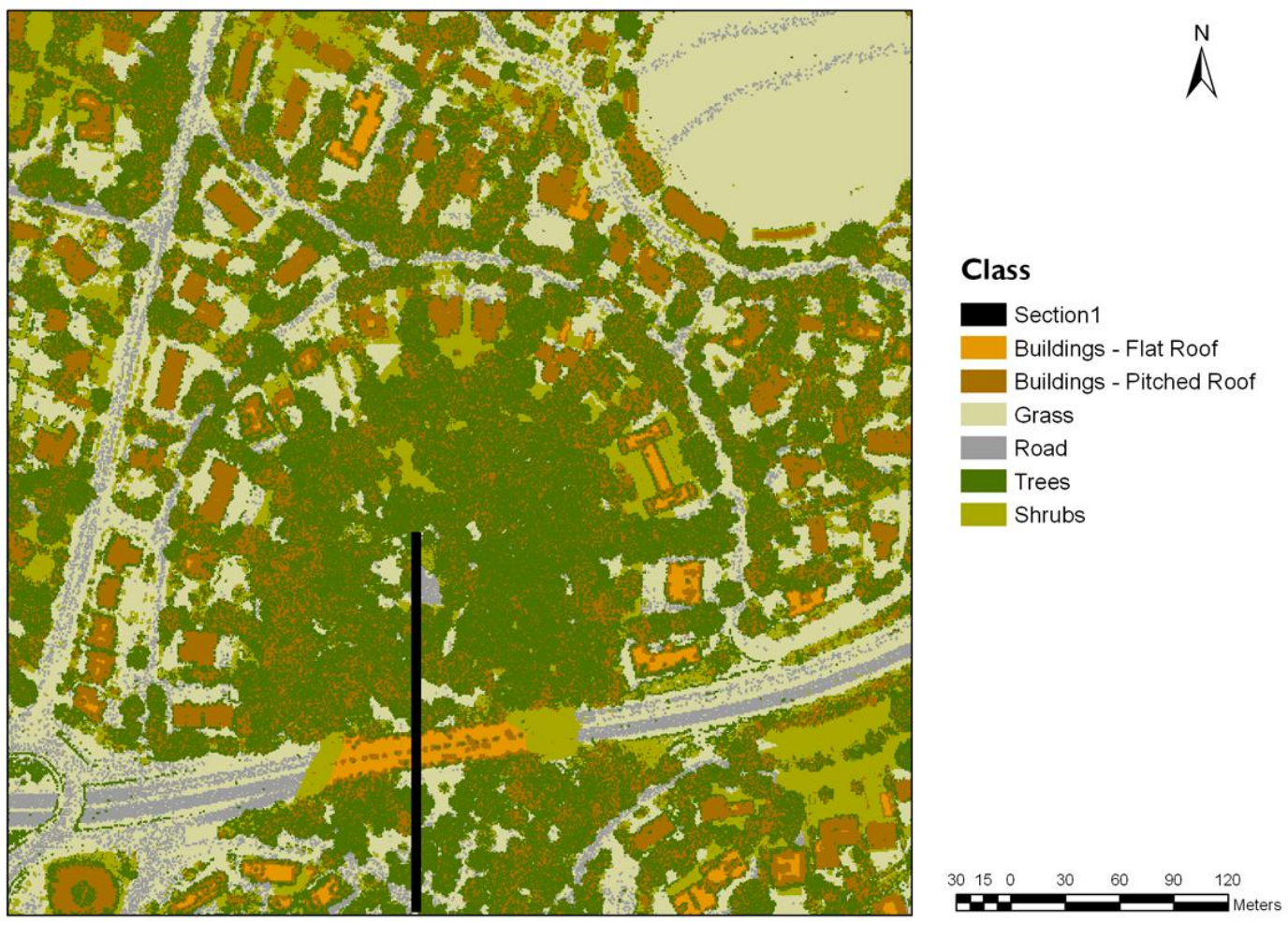

Figure 2: Points classified using the decision tree with amplitude as an attribute, converted to polygons by merging Thiessen polygons based on class. The points within the rectangle containing the misclassified road are shown in Figure 3.

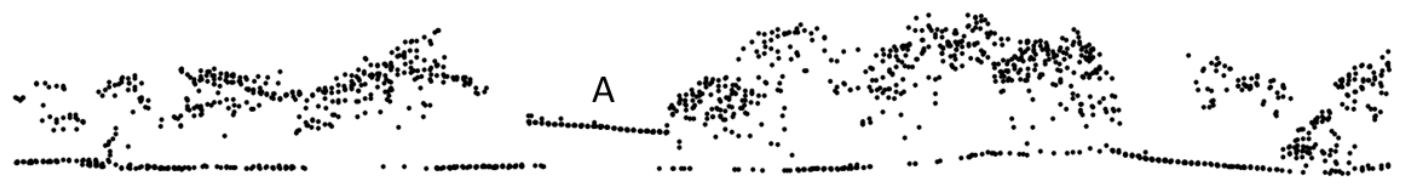

Figure 3: Section through the misclassified road (A). The section is through the rectangle shown in Figure 2.

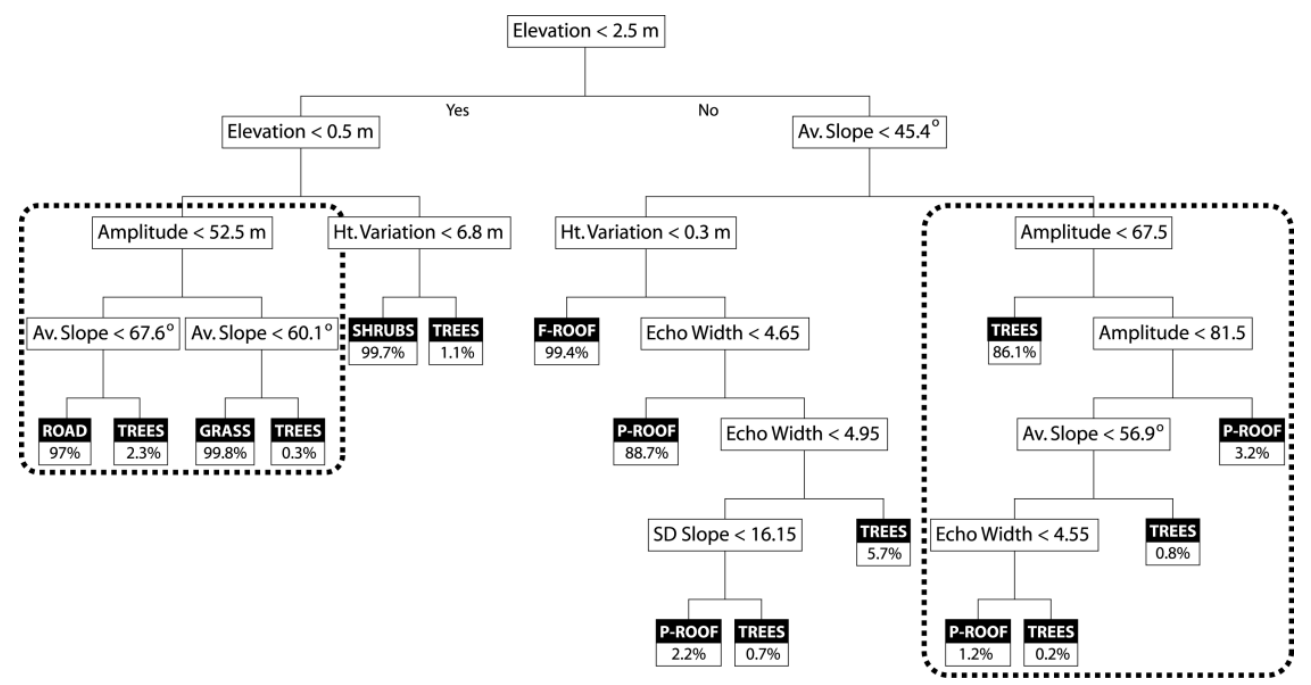

Figure 4: Nodes of the decision tree dependent on amplitude values for classification (within the rectangles)

After pruning, the decision tree using $\gamma$ as an attribute had 31 nodes including 16 leaf nodes. Grass, road and flat roofs required only one leaf node each (Figure 5). As in the decision tree using 
amplitude as an attribute, the greatest difficulty was in the separation of pitched roofs and trees. The classified points using the decision tree with $\gamma$ as an attribute are shown in Figure 6.

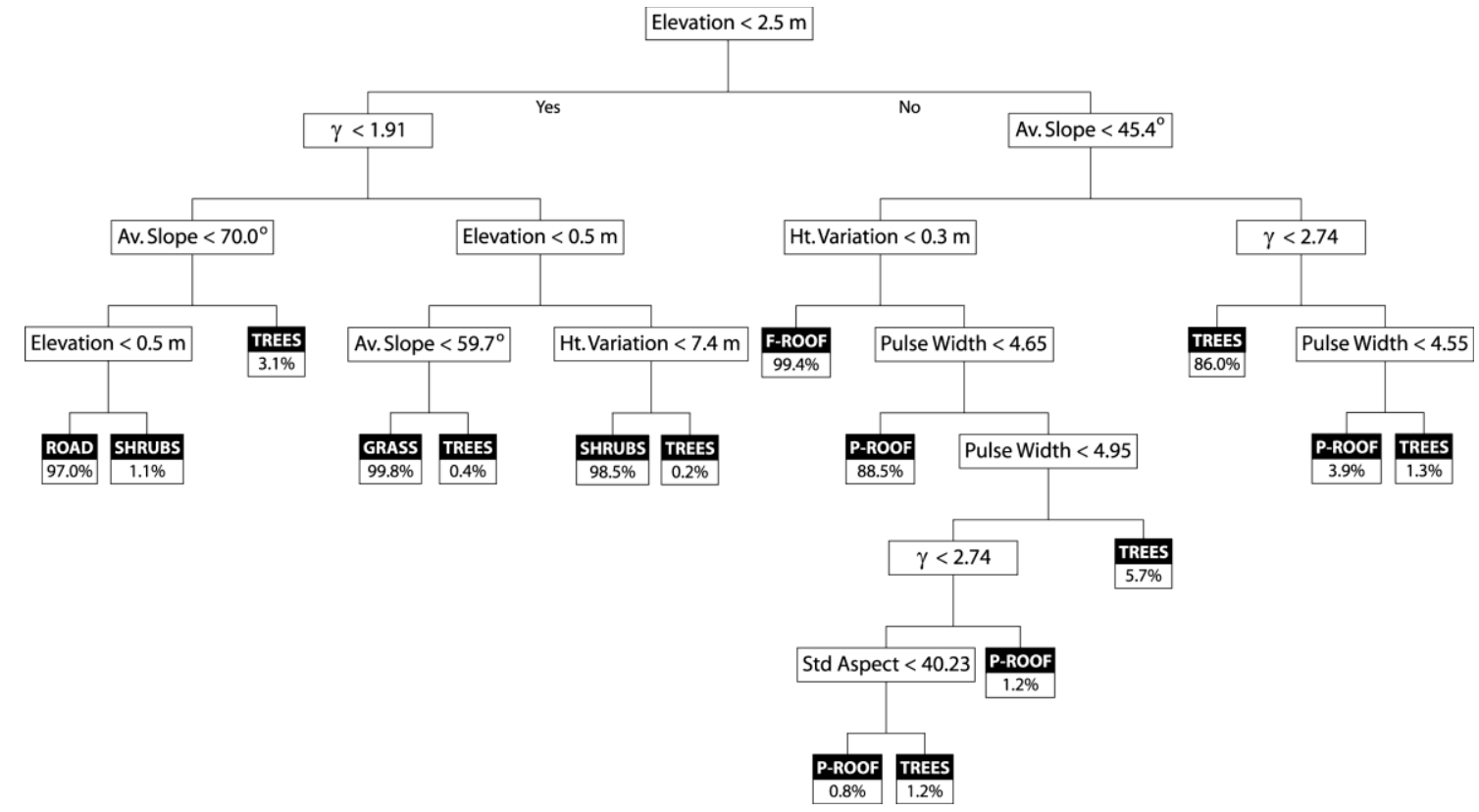

Figure 5: Decision tree with $\gamma$ as an attribute.
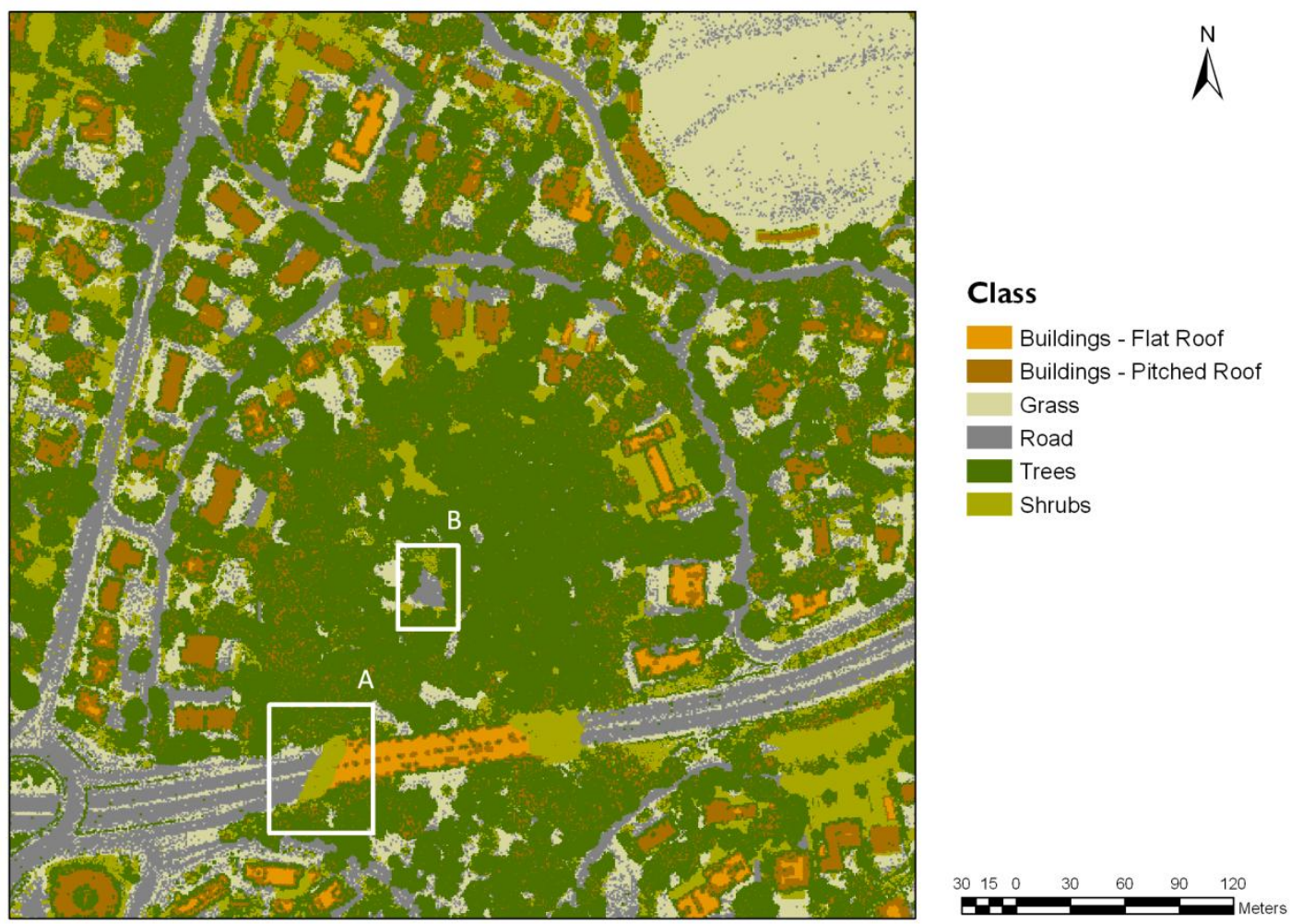

Figure 6: Points classified using the decision tree with the backscatter coefficient $(\gamma)$ as an attribute, converted to polygons by merging Thiessen polygons based on class. (A) Road incorrectly classified as shrubs, and (B) water body incorrectly classified as road. 


\section{Discussion}

\subsection{Comparison of the Calibration Constants}

The calibration constant in Bournemouth is nearly four times the calibration constant in Bristol. As noted in section 2, the average flying heights, in Bristol and Bournemouth, were $950 \mathrm{~m}$ and $300 \mathrm{~m}$ respectively. This accounts for much of the difference in the values of the calibration constants in the two study areas, as shown in equation [7]. From equation [3], the calibration constant is derived from the divergence angle of the laser beam, range, amplitude, and echo width of each point on the selected sections of asphalt road. The divergence angle of the laser beam is the same for the two study areas as the same sensor is used in both the cases. The ratio of the mean range of the selected points in Bournemouth to the mean range in Bristol is approximately 2.85. Similarly, the ratios of the mean amplitude and echo width are approximately 0.48 and 0.98 respectively. Therefore, the ratio of the calibration constants in Bristol, $C_{c a l 1}$, and Bournemouth, $C_{c a l 2}$, is:

$$
\begin{gathered}
C_{\text {cal1 }}=\frac{0.25 \pi \beta^{2}}{\mathrm{R}_{1}^{2} \cdot P_{1} \cdot W_{1}} ; \quad C_{\text {cal } 2}=\frac{0.25 \pi \beta^{2}}{\mathrm{R}_{2}^{2} \cdot P_{2} \cdot W_{2}} \\
\frac{C_{\text {cal2 }}}{C_{\text {cal1 }}}=\frac{\mathrm{R}_{1}^{2} \cdot P_{1} \cdot W_{1}}{\mathrm{R}_{2}^{2} \cdot P_{2} \cdot W_{2}}=\left(\frac{\mathrm{R}_{1}}{\mathrm{R}_{2}}\right)^{2} \cdot \frac{P_{1}}{P_{2}} \cdot \frac{W_{1}}{W_{2}}=2.85^{2} \cdot 0.48 \cdot 0.98=3.82
\end{gathered}
$$

where $\beta$ is the laser beam divergence angle, and $P_{1}$ and $P_{2}$ are the average amplitudes and $W_{1}$ and $W_{2}$ are the average echo widths of points on the selected sections of asphalt road in Bristol and Bournemouth.

The road sections were selected to have scan angles close to $90^{\circ}$ to reduce the effects of scan angles on the amplitude and echo width. Even then, there were slight differences in the calculated calibration constants within each study area. The means of the calibration constants for all the points in the selected sections of road in both the study areas were used for the analysis. The mean of the calculated calibration constants in Bristol was found to be $4.45 \times 10^{-6}$, when the average of all the points was calculated. When the means of the sections were calculated, and the calibration constant was taken as the mean of these means, a value of $4.84 \times 10^{-6}$ was obtained. The slight differences in the values could be due to the differences in the age and material used for surfacing the roads, leading to differences in reflectance values. This could have an impact on the calibration constant, and thereby on the classification. Similarly, the mean of the calculated calibration constants in Bournemouth was found to be $17.38 \times 10^{-6}$, when the mean of all the points was calculated. When the means of the sections were calculated, and the calibration constant was taken as the mean of these means, a value of $16.86 \times 10^{-6}$ was obtained.

\subsection{Accuracies of Classification}

The three decision trees based on amplitude, $\sigma$ and $\gamma$ had different numbers of nodes after pruning. Since the decision tree using amplitude as an attribute contained 31 nodes, the two other decision trees were pruned further to have a maximum of 31 nodes for a better comparison. Points from no other class are classified as flat roof using any of the attributes. Their user's accuracies are therefore $100 \%$.

The average user's and producer's accuracies are the highest for the classification using $\gamma$, and the accuracies for all the classes are above $70 \%$ (Table 2). The decision tree using $\gamma$ made use of five additional attributes - classified normalised elevation, height variation, average slope of TIN 
triangles attached to each point, echo width and standard deviation of aspects of TIN triangles to provide an overall accuracy of $91.5 \%$ and a kappa coefficient of 0.89 . Ten of the nodes in the decision tree using $\gamma$ required only three attributes for classification. Five nodes required four attributes, and one node required all six attributes.

Some of the points classified as shrubs and within roads, were classified incorrectly due to their estimated elevations from the ground. Some of the other points incorrectly classified as shrubs are from vehicles, which would also have a normalised elevation greater than $0.5 \mathrm{~m}$. Water has low reflectance in the infrared wavelengths, leading to the inland water body being classified as road (Figure 6). Flat roofs were classified based only on height, average slope and height variation. Grass and road were classified based on height and $\gamma$. Average slope was used only to filter out points from trees in both grass and road. Echo width and $\gamma$ are used to separate trees and pitched roofs. The values for echo width are generally larger for trees than pitched roofs.

The classification using $\sigma$ gave the highest number of correctly classified trees. It would also seem from Table 1 that the accuracies are comparable to the one using amplitude. However, it can be seen from Table 2 that all the points from grass are incorrectly classified using $\sigma$. The majority of them are classified as road. Although the producer's accuracies for the classification using $\sigma$ are the highest for trees, shrubs and road, the accuracies for grass are $0 \%$. It would therefore be better not to use this attribute for classification when all the classes are considered.

Table 2: Error matrix showing points in each class in the reference data and from the decision tree classifications using amplitude, backscatter cross section $(\sigma)$ and backscatter coefficient $(\gamma)$. User's and producer's accuracies of classifications for the three decision trees are also shown.

\begin{tabular}{|c|c|c|c|c|c|c|c|c|}
\hline & \multicolumn{8}{|c|}{ Classified Points } \\
\hline & Reference Points & Trees & Shrubs & Grass & Road & F-Roof & P-Roof & Producer's Accuracy \\
\hline \multirow[t]{7}{*}{ Amplitude } & Trees & 625 & 0 & 0 & 1 & 0 & 450 & 58.09 \\
\hline & Shrubs & 4 & 105 & 0 & 0 & 0 & 0 & 96.33 \\
\hline & Grass & 1 & 1 & 705 & 1 & 0 & 0 & 99.58 \\
\hline & Road & 0 & 0 & 361 & 341 & 0 & 0 & 48.58 \\
\hline & F-Roof & 7 & 23 & 27 & 3 & 284 & 55 & 71.18 \\
\hline & P-Roof & 1 & 0 & 0 & 0 & 0 & 525 & 99.81 \\
\hline & User's Accuracy & 97.96 & 81.40 & 64.50 & 98.55 & 100.00 & 50.97 & \\
\hline \multirow[t]{7}{*}{$\sigma$} & Trees & 1029 & 3 & 0 & 2 & 0 & 42 & 95.63 \\
\hline & Shrubs & 2 & 107 & 0 & 0 & 0 & 0 & 98.17 \\
\hline & Grass & 0 & 1 & 0 & 707 & 0 & 0 & 0.00 \\
\hline & Road & 0 & 0 & 0 & 702 & 0 & 0 & 100.00 \\
\hline & F-Roof & 13 & 23 & 0 & 30 & 284 & 49 & 71.18 \\
\hline & P-Roof & 25 & 0 & 0 & 0 & 0 & 501 & 95.25 \\
\hline & User's Accuracy & 96.26 & 79.85 & 0.00 & 48.72 & 100.00 & 84.63 & \\
\hline \multirow[t]{7}{*}{$\gamma$} & Trees & 973 & 1 & 0 & 3 & 0 & 99 & 90.43 \\
\hline & Shrubs & 4 & 105 & 0 & 0 & 0 & 0 & 96.33 \\
\hline & Grass & 1 & 1 & 669 & 37 & 0 & 0 & 94.49 \\
\hline & Road & 0 & 0 & 27 & 675 & 0 & 0 & 96.15 \\
\hline & F-Roof & 11 & 23 & 23 & 7 & 284 & 51 & 71.18 \\
\hline & P-Roof & 10 & 0 & 0 & 0 & 0 & 516 & 98.10 \\
\hline & User's Accuracy & 97.40 & 80.77 & 93.05 & 93.49 & 100.00 & 77.48 & \\
\hline
\end{tabular}


The classification using amplitude was compared with the one using $\gamma$ (Table 3). The values representing the incorrectly classified points, which are more than $10 \%$ of the total number, are underlined in the table. There are 50251 points that are classified as pitched roofs using amplitude and trees using $\gamma$, of which only $3848(7.6 \%)$ points are within the building polygons from OS MasterMap. The majority can therefore be considered to be correctly classified using $\gamma$.

26135 points are classified as grass using amplitude and road using $\gamma$ of which 15215 (58.2\%) points are within the polygons classified as road or track, roadside or path. Again, the majority of them are correctly classified using $\gamma$. There are 3098 points that are classified as shrubs using amplitude and trees using $\gamma$. These misclassifications could be less important, as they are classified as vegetation in both the classifications.

Table 3: Comparison of classifications using decision trees with amplitude and $\gamma$ as attributes

\begin{tabular}{|l|l|l|l|l|l|l|l|}
\hline \multirow{3}{*}{$\begin{array}{l}\text { Amplitude } \\
\text { Trees }\end{array}$} & \multicolumn{7}{|c|}{ Backscatter Coefficient } \\
\cline { 2 - 7 } Shrubs & Trees & Shrubs & Grass & Road & F-Roof & P-Roof & Total \\
Grass & $\underline{3098}$ & 1676 & 0 & 1792 & 0 & 270 & 144556 \\
Road & 77 & 0 & 0 & 0 & 0 & 0 & 22465 \\
F-Roof & 1 & 0 & 36099 & $\underline{26135}$ & 0 & 0 & 75311 \\
P-Roof & 0 & 0 & 0 & 15848 & 0 & 0 & 15852 \\
Total & 50251 & 0 & 0 & 0 & 4162 & 0 & 4162 \\
\hline
\end{tabular}

\subsection{Analysis of the Attributes}

The training data in Bristol, and the manually classified points in Bournemouth, were used for further analysis of the attributes related to intensity. The training data contains 'purer' data than the manually classified points. For example, the training points from a building would not include points from the edges. In the manually classified points, they are included to get a better idea about the classification accuracy. This has to be taken into consideration when the attributes are analysed.

As noted earlier, grass, road, pitched roof and trees require intensity for their classification, and these classes are analysed in this section. The values of the echo amplitude for grass and road are more clearly separated in the Bristol data than in Bournemouth (Figure 7). There is considerable overlap between the amplitudes of pitched roofs and trees in Bournemouth.
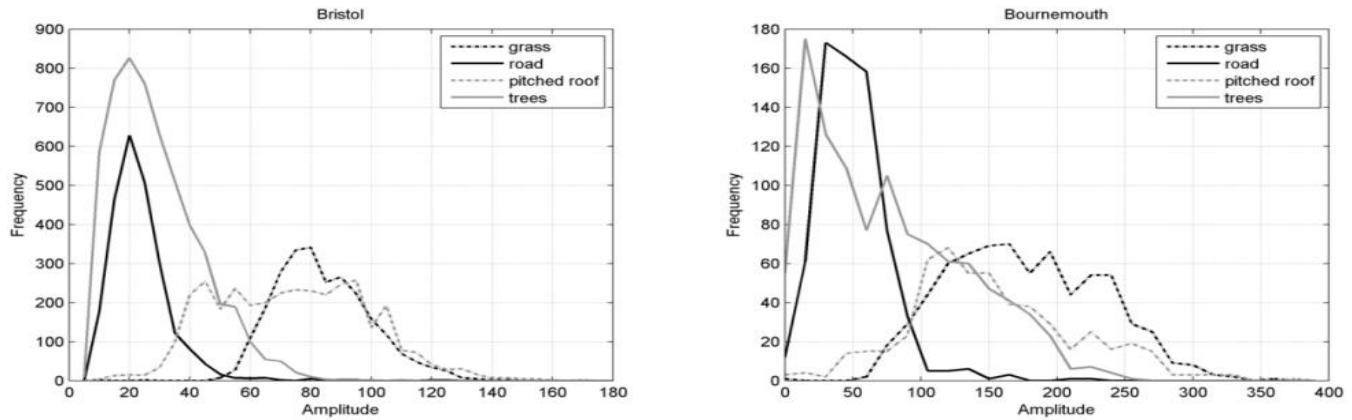

Figure 7: Frequency distribution of the amplitudes of echoes from grass, road, trees and pitched roofs in Bristol and Bournemouth

The values for amplitude and backscatter cross section seem to be very different for the two study areas (Figure 8). The amplitude values for grass and road are higher in Bournemouth than in Bristol. 
This is because of the differences in the altitudes from which the data were captured. The energy of the return signal is usually higher at a lower altitude, due to the reduction in the distance between the sensor and the target. The values for backscatter cross section are higher in Bristol than in Bournemouth, in contrast to the amplitudes. This could also be due to similar reasons as above. A higher flying height would mean a decrease in the energy of the return signal, and would require a larger area, or backscatter cross section, to represent the idealised scatterer, as described in section 1. The value of the backscatter cross section used to separate grass and road in the decision tree classifier is also shown in Figure 8. Since it was derived from the training dataset in Bristol, it seems to separate grass and road in Bristol quite well. However, all the values for grass in Bournemouth are less than this value. This accounts for the null accuracies for grass using backscatter cross section.
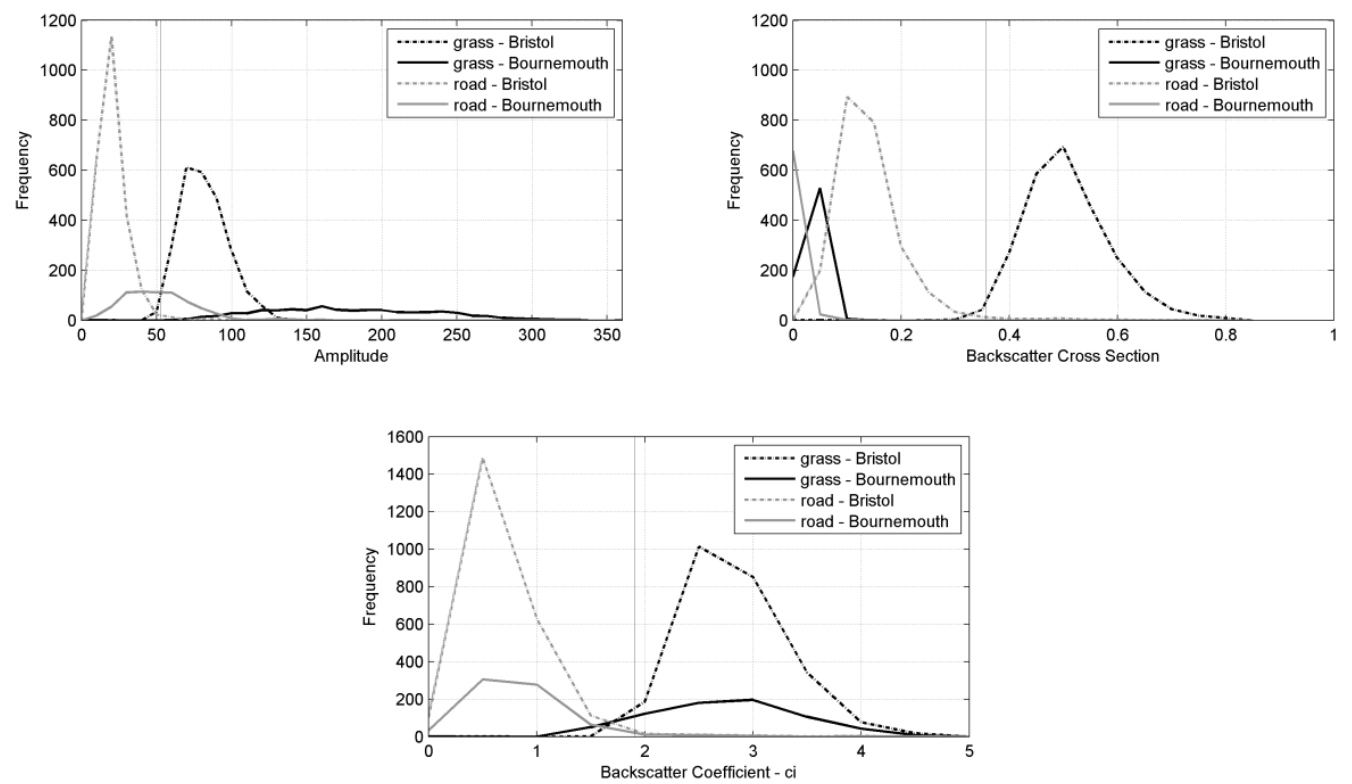

Figure 8: Frequency distribution of the amplitudes, backscatter cross sections and backscatter coefficients of echoes from grass and road in Bristol and Bournemouth. The values of the attributes used for separating grass and road in the decision tree classifiers are also shown.

The amplitude values for trees and pitched roofs are higher in Bournemouth than in Bristol (Figure 9). As seen in the case of grass and road, the backscatter cross sections are also higher in Bristol than in Bournemouth. The pitched roofs and trees can be differentiated better using $\gamma$. In the case of multiple echoes, the values for $\gamma$ are derived by dividing the backscatter cross section of each point with the area of the circular footprints, and not with the area of each individual target. Therefore, there might be a difference between the values of $\gamma$ for single and multiple echoes in the case of trees. 

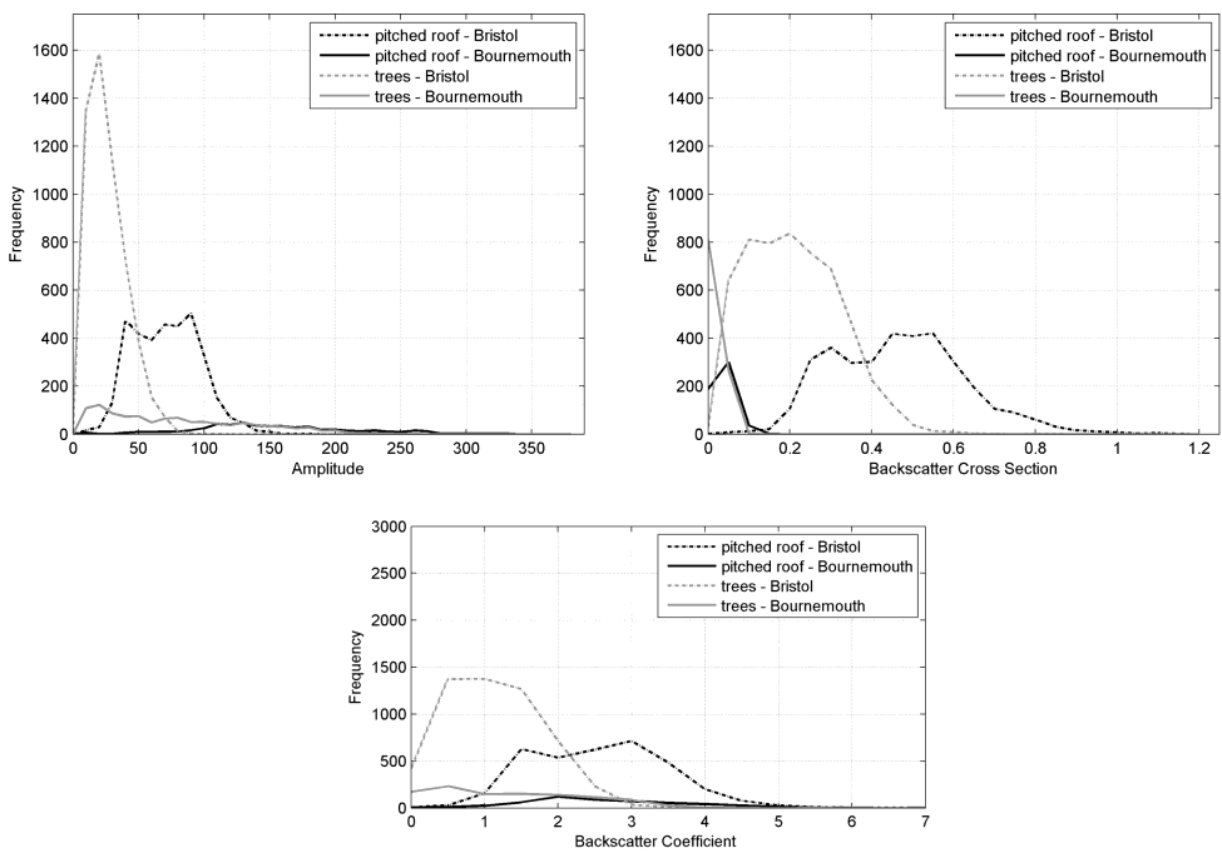

Figure 9: Frequency distribution of the amplitudes, backscatter cross sections backscatter coefficients of echoes from pitched roofs and trees in Bristol and Bournemouth

This was analysed further by separating the single echoes and the multiple echoes from trees in Bournemouth. In $\gamma$, the multiple echoes from trees can be differentiated more easily than single echoes when compared with pitched roofs. This probably accounts for the incorrect classifications of trees as building roofs. This can also be seen from the values of the backscatter coefficients used in the decision trees (Figure 10).

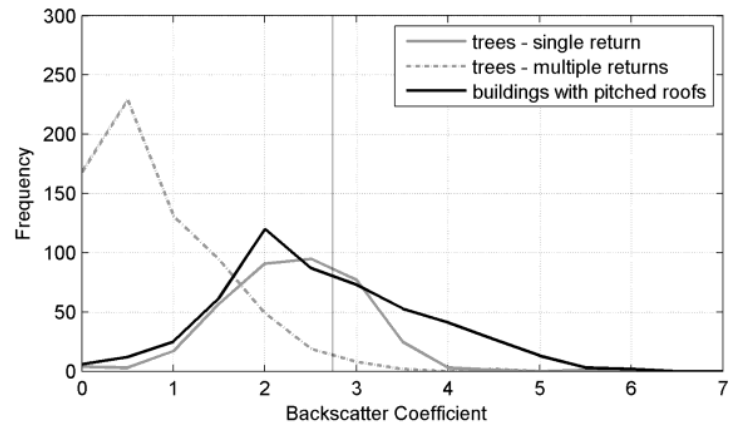

Figure 10: Backscatter coefficients of pitched roofs and trees in Bournemouth. The single and multiple echoes from trees are shown separately. The values of the attributes used for separating trees and pitched roofs in the decision tree classifiers are also shown.

\section{Conclusion}

This study suggests that full-waveform data could be more useful than discrete return data for topographic mapping as intensity is a very useful attribute in classification, and calibration of intensity is currently possible only with full-waveform data. As the amplitude values are dependent on various factors such as flying height, scan angle and weather conditions, calibrated intensity might be necessary to make any classification method transferable. Backscatter coefficient, derived from echo amplitude, width and range, proved more useful than amplitude and backscatter cross 
section in a decision tree classifier, when it has to be used on different datasets with differing flying heights and maximum scan angles, especially for natural and artificial ground.

The reflectance of asphalt chosen for calculating the calibration constant could have an influence on the results of the classifications. The calibration constants for both the datasets were calculated assuming the reflectance of asphalt to be 0.25 from Briese et al. (2008). However, the fact sheet for the scanning system gives this value as 0.20 . From equation [7], the chosen values did not make a difference to the ratio of the calibration constants, as long as they were the same for the two sites. The classification can probably be improved by on-site calibration before or after data capture. The calculation of normalised elevation is dependent on the accuracy of the DTM. While a rough DTM may be sufficient for an area with flat terrain, in complicated areas with bridges and flyovers, as in the study area in Bournemouth, a more accurate DTM might produce a better classification.

\section{Acknowledgements}

This study was conducted with financial support and data from Ordnance Survey, Southampton, and a scholarship from the University of Leicester. We would like to acknowledge the support from Dr. Sarah Smith-Voysey (Ordnance Survey) for the project. We are grateful to Mr. Hubert Lehner (Vienna University of Technology) for helpful discussions.

\section{References}

Alexander, C., Tansey, K., Tate, N.J., Smith-Voysey, S. and Kaduk, J., 2008. Extraction of Vegetation for Topographic Mapping from Full-waveform Airborne Laser Scanning Data. Silvilaser 2008: 8th international conference on LiDAR applications in forest assessment and inventory: 343353.

Bretar, F., Chauve, A., Bailly, J.-S., Mallet, C. and Jacome, A., 2009. Terrain surfaces and 3-D landcover classification from small footprint full-waveform lidar data: application to badlands. Hydrology and Earth System Sciences, 13 (8): 1531-1544.

Briese, C., Höfle, B., Lehner, H., Wagner, W., Pfennigbauer, M. and Ullrich, A., 2008. Calibration of Full-waveform Airborne Laser Scanning Data for Object Classification. Proceedings of SPIE The International Society for Optical Engineering, 6950: 69500H.

Coren, F. and Sterzai, P., 2006. Radiometric Correction in Laser Scanning. International Journal of Remote Sensing, 27(15): 3097-3104.

Ducic, V., Hollaus, M., Ullrich, A., Wagner, W. and Melzer, T., 2006. 3D Vegetation Mapping and Classification Using Full-Waveform Laser Scanning. Workshop on 3D Remote Sensing in Forestry, Vienna: 211-217.

Höfle, B. and Pfeifer, N., 2007. Correction of Laser Scanning Intensity Data: Data and Model-driven Approaches. ISPRS Journal of Photogrammetry and Remote Sensing, 62: 415-433.

Hofton, M.A., Minster, J.B. and Blair, J.B., 2000. Decomposition of Laser Altimeter Waveforms. IEEE Transactions on Geoscience and Remote Sensing, 38(4): 1989-1996.

Jelalian, A.V., 1991. Laser Radar Systems. Artech House Radar Library.

Kaasalainen, S., Hyyppa, J., Litkey, P., Hyyppa, H., Ahokas, E., Kukko, A. and Kaartinen, H., 2007. Radiometric calibration of ALS intensity. International Archives of Photogrammetry, Remote Sensing, and Spatial Information Sciences, 36, Part 3/W52: 201-205.

Mallet, C. and Bretar, F., 2009. Full-waveform Topographic Lidar: State-of-the-art. ISPRS Journal of Photogrammetry and Remote Sensing, 64(1): 1-16. 
Mallet, C., Soergel, U. and Bretar, F., 2008. Analysis of Full-Waveform LIDAR Data for an Accurate Classification of Urban Areas. ISPRS Congress, Proceedings of Commission III, Beijing 2008, 37-B3a: 85-91.

Quest, G.S.L., 2009. 3D Datum Transformations for Great Britain and Ireland.

Rutzinger, M., Höfle, B., Hollaus, M. and Pfeifer, N., 2008. Object-based Point Cloud Analysis of Fullwaveform Airborne Laser Scanning Data for Urban Vegetation Classification. Sensors, 8(8): 4505-4528

Schanda, E., 1986. Physical Fundamentals of Remote Sensing. Springer Verlag.

Ulaby, F.T., Moore, R.K. and Fung, A.K., 1982. Microwave Remote Sensing, Active and Passive Vol. 2. Artech House, Norwood, MA.

Wagner, W., Hollaus, M., Briese, C. and Ducic, V., 2008a. 3D Vegetation Mapping using Smallfootprint Full-waveform Airborne Laser Scanners. International Journal of Remote Sensing, 29(5): 1433-1452.

Wagner, W., Hyyppa, J., Ullrich, A., Lehner, H., Briese, C. and Kaasalainen, S., 2008b. Radiometric Calibration of Full-waveform Small-footprint Airborne Laser Scanners. The International Archives of the Photogrammetry, Remote Sensing and Spatial Information Sciences, XXXVII: 163-168.

Wagner, W., Ullrich, A., Ducic, V., Melzer, T. and Studnicka, N., 2006. Gaussian Decomposition and Calibration of a Novel Small-footprint Full-waveform Digitising Airborne Laser Scanner. ISPRS Journal of Photogrammetry and Remote Sensing, 60: 100-112.

Weisstein, E.W., 2009. Full Width at Half Maximum. http://mathworld.wolfram.com/FullWidthatHalfMaximum.html, 2009(14-08).

Woodhouse, I., 2006. Introduction to Microwave Remote Sensing. Taylor \& Francis, New York. Yongwei, S., 2008. Quantifying the Size of a Lidar Footprint: A Set of Generalized Equations. IEEE Geoscience and Remote Sensing Letters, 5(3): 419-422. 